\title{
REVISÃo de LITERATURA O insight no transtorno bipolar: uma revisão sistemática
}

\author{
Insight in bipolar disorder: a systematic review
}

Rafael de Assis da Silva ${ }^{1,2}$, Daniel C. Mograbi3,4, J. Landeira-Fernandez ${ }^{3,5}$, Elie Cheniaux ${ }^{2,6}$

\section{RESUMO}

Objetivos: Realizar uma revisão sistemática para compreender que fatores estão relacionados a uma maior ou menor consciência de morbidade no transtorno bipolar (TB), como o insight varia em função do estado afetivo e estabelecer uma comparação com outros transtornos mentais. Métodos: Realizou-se uma revisão sistemática da literatura científica sobre $\mathrm{o}$ insight em pacientes com TB. Foram buscados estudos clínicos originais sobre o tema nas bases de dados Medline, ISI e SciELO. Os termos de busca empregados foram: "insight" OR "awareness" AND "bipolar" OR "mania" OR "manic". Resultados: Foram selecionados 55 artigos. O insight no TB parece ser mais prejudicado do que na depressão unipolar, porém menos do que na esquizofrenia. Com relação ao TB, um menor nível de insight está relacionado à presença de sintomas psicóticos e de alterações cognitivas. Além disso, um comprometimento do insight está associado a uma menor adesão ao tratamento. Por outro lado, uma maior preservação do insight pode estar associada a maior ideação suicida. Finalmente, a fase maníaca cursa com um nível inferior de insight quando comparada à fase depressiva ou de

\section{Palavras-chave}

Insight, transtorno bipolar, mania, humor.

\section{Keywords}

Insight, bipolar disorder, mania, mood. eutimia. Conclusão: No TB, o insight está significativamente prejudicado, especialmente na mania. Diversos fatores clínicos parecem influenciar o nível de insight.

\section{ABSTRACT}

Objectives: To conduct a systematic review to understand which factors are related to insight in bipolar disorder (BD), how insight varies depending on affective state and to establish a comparison between BD and other mental disorders. Methods: We performed a systematic review of the scientific literature on insight in $\mathrm{BD}$ patients. Clinical studies on the topic were searched in the Medline, SciELO and ISI databases. The search terms used were: "insight" OR "Awareness" AND "bipolar" OR "mania". Results: Fifty-five articles were selected. Insight in BD is more compromised than in unipolar depression, but less than in schizophrenia. A lower level of insight is related to the presence of psychotic symptoms and cognitive disorders. Moreover, insight impairment is associated with lower adherence to treatment. On the other hand, a greater preservation of insight may be associated with increased suicidal ideation. Finally, the manic phase is characterized by a lower level of insight than the depressive phase or euthymia. Conclusion: Insight is greatly impaired in BD, especially during mania. Several clinical factors seem to influence the level of insight.

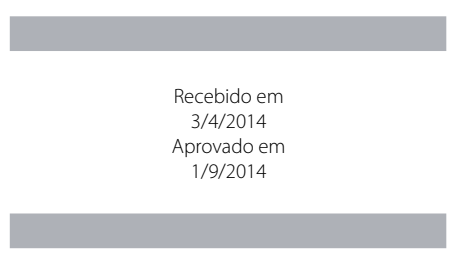

DOI: $10.1590 / 0047-2085000000032$
1 Instituto Municipal Philippe Pinel (IMPP).

2 Universidade Federal do Rio de Janeiro, Instituto de Psiquiatria (UFRJ/Ipub).

3 Pontifícia Universidade Católica do Rio de Janeiro (PUC-Rio).

4 Institute of Psychiatry, King's College London, UK.

5 Universidade Estácio de Sá (Unesa).

6 Universidade do Estado do Rio de Janeiro, Faculdade de Ciências Médicas (UERJ/FCM).

Endereço para correspondência: Rafael de Assis da Silva

Rua Largo do Machado, 29/411, Catete

20751-430 - Rio de Janeiro, RJ, Brasil

E-mail: rafaelpsiq@gmail.com 


\section{INTRODUÇÃO}

O insight tradicionalmente era definido como uma "correta atitude para mudanças mórbidas em si mesmo'". Uma menor ou maior consciência quanto a estar doente ou apresentar sintomas ou algum prejuízo psicossocial pode influenciar significativamente a evolução da própria doença, afetando no mínimo a adesão ao tratamento ${ }^{2-5}$.

O insight é investigado em pacientes neurológicos desde o final do século XIX, passando a ser pesquisado em pacientes psiquiátricos apenas mais recentemente. Inicialmente, muitos estudos sobre esse aspecto foram conduzidos com pacientes esquizofrênicos ${ }^{6-10}$. Contudo, nos últimos anos, o insight em pacientes com transtorno bipolar (TB) passou a ser estudado também. Grande parte dos pacientes bipolares mostra déficits em sua consciência sobre estar doente ou em sua consciência sobre sinais ou sintomas específicos ${ }^{11}$.

A falta de insight no TB pode ser bastante prejudicial para a formulação adequada desse diagnóstico. A observação clínica tem indicado que o insight está mais comprometido na mania do que na depressão $0^{12-14}$. Nesse sentido, pacientes com TB com frequência não relatam seus sintomas ou episódios maníacos, sendo, assim, erroneamente diagnosticados com depressão unipolar ${ }^{15,16}$.

Algumas questões sobre o insight no TB parecem ainda não estar claras. A experiência clínica mostra que maior nível educacional poderia ser um fator favorável a um melhor insight ${ }^{17}$. Nessa linha de raciocínio, é plausível pensar que maior conhecimento sobre a doença e sobre sua sintomatologia poderia exercer papel importante sobre melhor nível de insight. Entretanto Güçlü et al. ${ }^{18}$ não encontraram correlação entre maior nível educacional e melhor nível de insight em TB. Além disso, possivelmente maior tempo de doença ou número maior de internações ou de episódios afetivos poderia conferir ao indivíduo bipolar maior conhecimento sobre a doença e, consequentemente, maior insight. Por outro lado, a associação entre outros fatores sociodemográficos, como gênero ou idade, e o insight parece não ter sido amplamente estudada. Também se torna importante discutir outras possíveis consequências do comprometimento do insight em bipolares, além do prejuízo da adesão medicamentosa $a^{2-5}$. A possível influência do insight sobre a qualidade de vida ou sobre a evolução da doença, incluindo tentativas de suicídio, precisa ser mais bem compreendida.

Outra questão importante diz respeito à influência de alterações neurocognitivas sobre o insight. Estudo com pacientes com demência mostra que um comprometimento cognitivo está associado a pior insight ${ }^{19}$. É de conhecimento dos especialistas que pacientes com TB podem cursar com comprometimento cognitivo ${ }^{20,21}$. Os estudos sobre a associação entre alterações cognitivas e insight no TB são ainda pouco numerosos.
Em um estudo anterior ${ }^{14}$, observamos que, no $T B$, os pacientes em mania se autoavaliaram de forma semelhante àqueles em eutimia. Em contraste, os indivíduos em depressão se autoavaliaram de forma distinta em relação aos que estavam em eutimia ou mania. Tais resultados poderiam ser explicados pelo fato de, na mania, o insight estar mais prejudicado do que nas outras fases do TB. Contudo, essa conclusão precisa ser corroborada por meio de estudos que avaliem o insight nos diferentes estados de humor encontrados nesse transtorno mental.

Outra questão relevante é a comparação, quanto ao nível de insight, do TB com outros transtornos mentais. Pini et al..22 e Colis et al. ${ }^{23}$ relatam que o insight de pacientes com TB é semelhante ao de pacientes esquizofrênicos. Contudo, outros estudos ${ }^{24,25}$ observaram que o insight em pacientes com TB era maior do que em esquizofrênicos. Também se torna importante discutir se a presença de sintomas psicóticos em fases agudas do TB pode influenciar o nível de insight. Além disso, também não está claro como o insight no TB se diferencia na comparação com outros transtornos mentais, como transtorno esquizoafetivo, depressão unipolar e transtornos de ansiedade.

Diante de tantas questões sobre o insight no TB e da relevância do tema, realizamos uma revisão sistemática de estudos sobre o insight no TB, com o objetivo de compreender as seguintes questões:

a) Que elementos sociodemográficos e clínicos estão associados ao nível de insight no TB?

b) Como o insight varia em função do estado afetivo de humor no TB?

c) Como o insight no TB se compara com outros transtornos mentais em relação ao insight?

\section{MÉTODOS}

Realizou-se uma revisão sistemática da literatura científica sobre o insight em pacientes com TB. Foram buscados estudos clínicos originais sobre o tema. Foram utilizadas as bases de dados Medline, ISI, e SciELO. Não houve restrição quanto ao período da publicação. Os termos de busca empregados foram: "insight" OR "awareness" AND "bipolar" OR "mania" OR "manic".

Os critérios de seleção dos artigos foram: 1. Estudos originais e empíricos. 2. Estudos publicados nas línguas inglesa, francesa, espanhola ou portuguesa. 3. Estudos em que o insight foi avaliado em uma amostra de pacientes com o diagnóstico de TB. 4. Os estudos que apresentassem amostras contendo outros tipos de transtornos mentais além do TB somente seriam incluídos se apresentassem resultados específicos para o grupo de pacientes bipolares. 5. Os estudos selecionados deveriam utilizar uma escala ou algum item específico que aferisse o insight. 6. As amostras deveriam ser 
constituídas somente por indivíduos adultos, com no mínimo dez pacientes.

Não houve procura por estudos não publicados. Somente um juiz realizou o julgamento dos artigos a serem incluídos/excluídos.

\section{RESULTADOS}

Na busca inicial, encontrou-se um total de 510 referências no Medline, 1.563 no ISI e 9 no SciELO, com diversas superposições entre essas bases de dados.

Após a leitura dos resumos das referências encontradas no Medline, selecionaram-se 62 artigos.

Quando se utiliza o termo "bipolar", diversos estudos sobre outras áreas de conhecimento que não a psiquiatria são selecionados. Esses estudos sobre outras áreas de conhecimento incluem artigos de pesquisa sobre nanotecnologia, engenharia e outras tecnologias, meteorologia, astronomia, eletroquímica, zoologia, entre outras. Pelo menos metade dos estudos selecionados nas bases de dados do Medline estava relacionada a alguma dessas áreas. Esse fato também ocorreu na pesquisa na base de dados ISI.

$\mathrm{Na}$ base ISI, após a leitura dos resumos dos artigos, selecionaram-se 58 artigos. Desses 58 artigos, 50 foram repetidos em relação à base Medline, portanto foram excluídos.

Na base SciELO, após a leitura dos resumos dos artigos, selecionaram-se dois artigos. Um desses artigos já havia sido citado na base ISI, portanto foi excluído.

Ao todo, foram selecionados 71 resumos das três bases pesquisadas. Esses 71 artigos foram lidos na íntegra. Um artigo foi excluído por apresentar amostra de bipolares adolescentes. Seis artigos foram excluídos por não apresentarem alguma escala específica de avaliação de insight. E nove artigos apresentaram amostras contendo outros tipos de transtornos mentais, além do TB, e não apresentaram resultados específicos para o grupo de pacientes bipolares, sendo, portanto, excluídos. Não foram encontrados artigos com menos de dez pacientes. Ao final, foram selecionados 55 artigos. O fluxograma de busca e seleção dos artigos aparece na figura 1.

\section{INSTRUMENTOS}

Os estudos foram bastante heterogêneos quanto aos instrumentos utilizados para avaliação do insight. Algumas escalas avaliaram itens específicos do insight. Esses itens encontram-se detalhados nas tabelas. As escalas mais utilizadas foram: a Scale of Unawareness of Mental Disorders (SUMD) ${ }^{26}$; o Schedule for Assessment of Insight (SAI) ${ }^{27}$ e sua versão estendida (SAl-E) 28; e o Insight and Treatment Attitudes Questionnaire $(\mathrm{ITAQ})^{7}$.

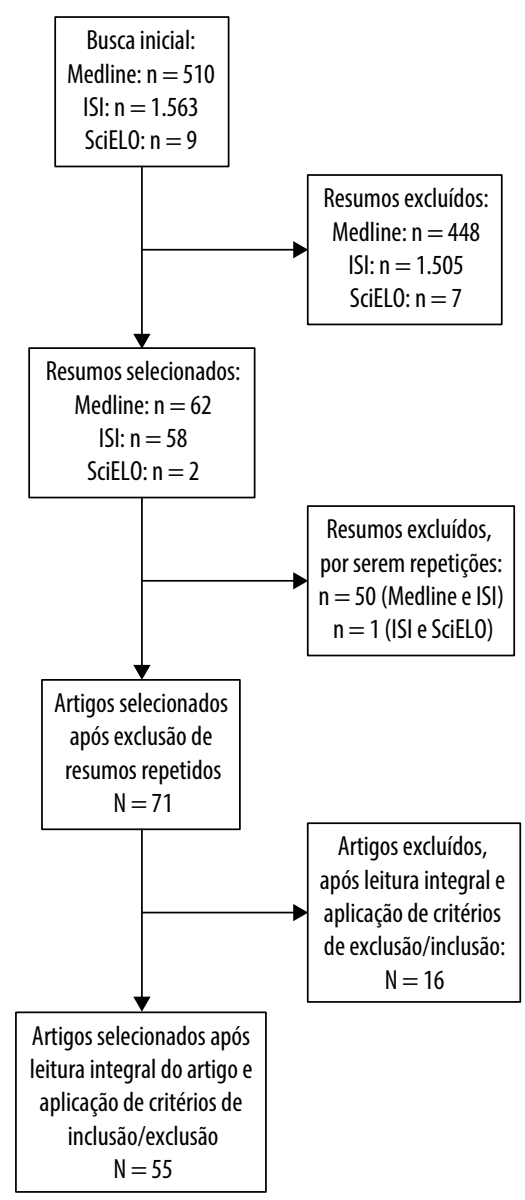

Figura 1. Fluxograma.

\section{Comparação entre o TB e outros transtornos mentais}

Os estudos sobre a comparação quanto ao insight entre o TB e outros transtornos mentais são apresentados na tabela 1.

Dezessete estudos ${ }^{11,22-25,29-40}$ compararam o insight entre esquizofrênicos e bipolares. Desses, 12 estudos 11,22,24,25,29-36 relataram maior nível de insight no TB do que na esquizofrenia, em pelo menos um dos subtipos de insight avaliado (ou seja, insight global, ou insight sobre a doença ou insight sobre o tratamento ou insight sobre as consequências sociais). Em contraste, cinco estudos ${ }^{11,23,37-40}$ não observaram diferença quanto ao nível de insight entre bipolares e esquizofrênicos. Em nenhum dos estudos o insight foi maior na esquizofrenia do que no TB.

Cinco estudos ${ }^{11,22,23,40,41}$ compararam o insight entre bipolares e esquizoafetivos. Desses, somente um $^{22}$ relatou maior insight sobre consequências sociais em esquizoafetivos do que em bipolares. Os outros quatro estudos ${ }^{11,23,40,41}$ não relataram qualquer diferença. Seis estudos $23,29,30,40-42$ compararam o insight entre deprimidos unipolares e bipolares. Desses, cinco estudos ${ }^{23,29,30,40,42}$ relataram que o insight no TB foi menor do que na depressão unipolar. Em contraste, um estu$\mathrm{do}^{41}$ relatou que o insight global de bipolares foi igual ao de deprimidos unipolares. Somente um estudo ${ }^{43}$ comparou o insight entre TB tipo I e TB tipo II, e observou que o insight global no TB do tipo I foi maior do que do tipo II. 
Tabela 1. Comparação entre o transtorno bipolar e outros transtornos mentais

\begin{tabular}{|c|c|c|c|c|}
\hline Estudo & Desenho & Tipo de insight & Instrumento de avaliação & Resultados \\
\hline Jonsdottir et al. (2013) & TB $(n=101)$ vs. SCH $(n=154)$ & Global & BIS & $\mathrm{TB}=\mathrm{SCH}$ \\
\hline Braw et al. (2012) & TB eu $(n=34)$ vs. SCH $(n=32)$ & $\begin{array}{l}\text { Sobre a doença e sobre consequências } \\
\text { sociais }\end{array}$ & SUMD e PANSS G12 & $\mathrm{TB}>\mathrm{SCH}$ \\
\hline Calatayud et al. (2012) & TB com psicose $(n=43)$ vs. SCH $(n=86)$ & Global & PANSS G12 & $\mathrm{TB}>\mathrm{SCH}$ \\
\hline Govil et al. (2008) & TB eu $(n=40)$ vs. SCH $(n=40)$ & Sobre a doença & SAI-E + SUMD & $\mathrm{TB}>\mathrm{SCH}$ \\
\hline \multirow[t]{2}{*}{ Varga et al. (2007) } & $\begin{array}{l}\text { TB ( } 5 \mathrm{mn} ; 10 \mathrm{dp} ; 17 \text { em remissão total; } 5 \text { em } \\
\text { remissão parcial) vs. SCH ( } \mathrm{n}=32)\end{array}$ & Global & SUMD & $\mathrm{TB}=\mathrm{SCH}$ \\
\hline & & Sobre sintomas & & $\mathrm{TB}>\mathrm{SCH}$ \\
\hline Colis et al. (2006) & $\begin{array}{l}\text { TB }(n=18 m n) \text { vs. TB }(n=16 m s) \text { vs. TB }(n= \\
18 \text { dp) vs. SCHA }(n=30) \text { vs. SCH }(n=12) \text { vs. } \\
\text { MD }(n=56)\end{array}$ & Global & $\mathrm{BCIS}$ & $\mathrm{TB}=\mathrm{SCH}<\mathrm{MD} ; \mathrm{TB}=\mathrm{SCHA}$ \\
\hline \multirow[t]{6}{*}{ Pini et al. (2004) } & $\begin{array}{l}\text { TB }(\mathrm{n}=29, \mathrm{mn} \text { com psicose) vs. TB }(\mathrm{n}=49, \mathrm{~ms} \\
\text { com psicose) vs. SCH }(\mathrm{n}=46) \text { vs. SCHA }(\mathrm{n}=32)\end{array}$ & Sobre a doença & SUMD & $\mathrm{TB}=\mathrm{SCH}=\mathrm{SCHA}$ \\
\hline & & Sobre tratamento & & $\begin{array}{l}\mathrm{TB}(\mathrm{ms})>\mathrm{SCH} ; \mathrm{TB}(\mathrm{ms})=\mathrm{SCHA} ; \mathrm{TB} \\
(\mathrm{mn})=\mathrm{SCH} ; \mathrm{TB}(\mathrm{mn})=\mathrm{SCHA}\end{array}$ \\
\hline & & Sobre consequências sociais & & $\begin{array}{l}\mathrm{TB}(\mathrm{ms})>\mathrm{SCH} ; \mathrm{TB}(\mathrm{mn})<\mathrm{SCHA} ; \mathrm{TB} \\
(\mathrm{mn})=\mathrm{SCH} ; \mathrm{TB}(\mathrm{ms})=\mathrm{SCHA}\end{array}$ \\
\hline & & \multicolumn{2}{|c|}{ Sobre a doença referente a episódio afetivo prévio } & $\begin{array}{l}\mathrm{TB}(\mathrm{ms})>\mathrm{SCH} ; \mathrm{TB}(\mathrm{mn})>\mathrm{SCH} ; \mathrm{TB} \\
(\mathrm{ms})=\mathrm{SCHA} ; \mathrm{TB}(\mathrm{mn})=\mathrm{SCHA}\end{array}$ \\
\hline & & \multicolumn{2}{|l|}{ Sobre tratamento de episódio afetivo prévio } & $\begin{array}{l}\mathrm{TB}(\mathrm{ms})>\mathrm{SCH} ; \mathrm{SCH}<\mathrm{SCHA} ; \mathrm{TB} \\
(\mathrm{mn})=\mathrm{SCH} ; \mathrm{TB}(\mathrm{mn})=\mathrm{SCHA} ; \mathrm{TB} \\
(\mathrm{ms})=\mathrm{SCHA}\end{array}$ \\
\hline & & \multicolumn{2}{|c|}{ Sobre consequências sociais de episódio afetivo prévio } & $\begin{array}{l}\mathrm{TB}(\mathrm{ms})>\mathrm{SCH} ; \mathrm{TB}(\mathrm{mn})=\mathrm{SCHA} ; \mathrm{TB} \\
(\mathrm{mn})=\mathrm{SCH} ; \mathrm{TB}(\mathrm{ms})=\mathrm{SCHA}\end{array}$ \\
\hline Arduini et al. (2003) & $\mathrm{TB}(\mathrm{n}=22 \mathrm{mn}$ com psicose) vs. SCH $(\mathrm{n}=42)$ & Global & SUMD & $\mathrm{TB}=\mathrm{SCH}$ \\
\hline Daneluzzo et al. (2002) & $\begin{array}{l}\text { TB ( } 124 \mathrm{mn} \text {, com psicose; } 24 \mathrm{~ms} \text {, psicose) vs. } \\
\text { SCH ( }=86)\end{array}$ & Global & PANSS G12 & $\mathrm{TB}>\mathrm{SCH}$ \\
\hline Dell'Osso et al. (2002) & $\begin{array}{l}\text { TB com psicose ( } 55 \mathrm{mn} ; 62 \mathrm{~ms} ; 30 \mathrm{dp}) \text { vs. MD } \\
\text { com psicose }(\mathrm{n}=30)\end{array}$ & $\begin{array}{l}\text { Sobre a doença, tratamento e } \\
\text { consequências sociais }\end{array}$ & SUMD & $\mathrm{TB}<\mathrm{MD}$ \\
\hline Williams et al. (2002) & $T B(\mathrm{n}=33)$ vs. SCH $(\mathrm{n}=25)$ & Global & SAI & $\mathrm{TB}=\mathrm{SCH}$ \\
\hline Yen et al. (2002b) & $\begin{array}{l}\text { TB eu ( } n=33 \text {, com história de psicose; } n=32 \text {, } \\
\text { sem história de psicose) vs. } \mathrm{SCH}(\mathrm{n}=44)\end{array}$ & Global & SAl-E & $\begin{array}{l}\text { TB com psicose }=\text { SCH; TB sem } \\
\text { psicose }>\text { SCH }\end{array}$ \\
\hline Masson et al. (2001) & TB $(\mathrm{n}=18$, com psicose) vs. $\mathrm{SCH}(\mathrm{n}=37)$ & Sobre a doença & SUMD & $\mathrm{TB}>\mathrm{SCH}$ \\
\hline \multirow[t]{2}{*}{ Pini et al. (2001) } & $\begin{array}{l}\text { TB com psicose }(67 \mathrm{mn} ; 46 \mathrm{~ms} ; 37 \mathrm{dp}) \text { vs. SCH } \\
(\mathrm{n}=29) \text { vs. SCHA }(\mathrm{n}=24) \text { vs. MD ( }=30 \text {, com } \\
\text { psicose })\end{array}$ & Sobre a doença & SUMD & $\mathrm{TB}=\mathrm{SCH}$ \\
\hline & & \multicolumn{2}{|l|}{ Sobre tratamento (atual e retrospectivo) } & $\mathrm{TB}>\mathrm{SCH}$ \\
\hline Ghaemi et al. (2000) & $\begin{array}{l}\text { TB I }(\mathrm{n}=37) \text { vs. MD }(\mathrm{n}=34 \text { sem psicose; } \mathrm{n}= \\
\text { 5, com psicose) vs. } \mathrm{AD}(\mathrm{n}=13) \text { vs. TB II }(\mathrm{n}=8) \\
\text { vs. SCHA }(\mathrm{n}=3) \text { vs. } \mathrm{D}(\mathrm{n}=1)\end{array}$ & Global & SUMD & $\mathrm{TB}=\mathrm{MD}<\mathrm{AD}$ \\
\hline Weiler et al. (2000) & $\begin{array}{l}\text { TB }(n=40) \text { vs. SCH ( } n=81) \text { vs. MD }(n=33) \text { vs. } \\
\text { SCHA }(n=14) \text { vs. outros tr. psicóticos }(n=19)\end{array}$ & Global & ITAQ & $\mathrm{TB}<\mathrm{MD}$; $\mathrm{TB}>$ outros tr. psicóticos \\
\hline Pallanti et al. (1999) & $\begin{array}{l}\text { TB I }(n=25) \text { vs. TB II }(n=32) \text {; em remissão } \\
\text { total ou parcial }\end{array}$ & Global & SUMD & $\mathrm{TB} \|<\mathrm{TB}$ I \\
\hline Young et al. (1998) & $\mathrm{TB}(\mathrm{n}=21)$ vs. SCH $(\mathrm{n}=108)$ & Sobre doença e sobre sintomas & SUMD & $\mathrm{TB}>\mathrm{SCH}$ \\
\hline Fennig et al. (1996) & $\begin{array}{l}\text { TB }(n=52 ; \text { com psicose) vs. MD ( } n=35 ; \\
\text { com psicose) vs. SCH }(n=86) \text { vs. outros tr. } \\
\text { psicóticos }(n=16)\end{array}$ & Global & $\begin{array}{l}\text { Item modificado } \\
\text { de HDS }\end{array}$ & $\begin{array}{l}\text { outros tr. psicóticos }<\mathrm{SCH}<\mathrm{TB} \\
<\mathrm{MD}\end{array}$ \\
\hline Michalakeas et al. (1994) & TB $(\mathrm{n}=13 \mathrm{mn})$ vs. SCH $(\mathrm{n}=42)$ vs. $\mathrm{MD}(\mathrm{n}=22)$ & Global & ITAQ & $\mathrm{SCH}<\mathrm{TB}<\mathrm{MD}$ \\
\hline
\end{tabular}

TB: transtorno bipolar; mn: mania; ms: episódio misto; dp: depressão; eu: eutímico; SCH: esquizofrenia; SCHF: esquizofreniforme; MD: transtorno depressivo maior; TDP: transtorno delirante persistente; TPB: transtorno psicótico breve; SCHA: esquizoafetivo; AD: transtornos de ansiedade; D: distimia; NC: controles normais; SUMD: Scale of Unawareness of Mental Disorders; BCIS: Beck Cognitive Insight Scale; PANSS: Positive and Negative Syndrome Scale; HDS: Hamilton Depression Scale; ITAQ: Insight and Treatment Attitudes Questionnaire; <: menor insight que; >: maior insight que; = : sem diferenças quanto ao insight. 


\section{Dados sociodemográficos}

Os seis ${ }^{17,18,44-47}$ estudos que investigaram a associação entre insight e dados sociodemográficos são apresentados na tabela 2 .

Três estudos ${ }^{17,18,46}$ investigaram a relação entre insight e gênero. Desses, um estudo ${ }^{18}$ encontrou que o insight é menor no gênero feminino do que no masculino. Porém, um estudo ${ }^{46}$ encontrou o oposto e outro ${ }^{17}$ não encontrou correlação entre insight e gênero.

Quatro estudos 17,18,44,47 investigaram a relação entre insight e idade. Desses, dois estudos ${ }^{17,47}$ observaram que um menor insight está associado a idades mais elevadas. Dois outros estudos ${ }^{18,44}$, contudo, não encontraram correlação entre insight e idade. Por fim, três estudos ${ }^{17,45,47}$ de um total de quatro ${ }^{17,18,45,47}$ relataram que um menor insight estava correlacionado a um nível educacional mais baixo, e o outro estudo $^{18}$ não encontrou correlação entre nível educacional e insight.

\section{Delineamento}

\section{Dados clínicos retrospectivos}

Os $14^{17,18,23,44,46-55}$ estudos que investigaram a associação entre o insight e dados clínicos retrospectivos são apresentados na tabela 3.

Tabela 2. Dados sociodemográficos

\begin{tabular}{|c|c|c|c|c|}
\hline Estudo & Amostra & $\begin{array}{l}\text { Instrumento de } \\
\text { avaliação do insight }\end{array}$ & Variáveis & Resultados \\
\hline \multirow[t]{4}{*}{ Güçlü et al. (2011) } & 104 TB (mn ou ms) & SUMD & Insight e gênero & $<$ Insight: mulheres \\
\hline & & & Insight e idade & Sem correlação \\
\hline & & & Insight e nível educacional & Sem correlação \\
\hline & & & Insight retrospectivo sobre a doença e ocupação & $<$ Insight: desempregados \\
\hline Cassidy (2010) & 156 TB (86 mn, 29 ms; 14 dp, 27 eu) & Item 13 SMS & Insight e idade & Sem correlação \\
\hline \multirow[t]{3}{*}{ Dias et al. (2008b) } & $50 \mathrm{~TB}(\mathrm{eu})$ & SUMD & Insight (global e sobre tratamento) e idade & $<$ Insight: > idade \\
\hline & & & Insight (global e sobre tratamento) e nível educacional & $<$ Insight: $<$ nível educacional \\
\hline & & & Insight e gênero & Sem correlação \\
\hline Yen et al. (2008a) & 96 TB (eu) & SAl e SAI-E & Insight e nível educacional & $<$ Insight: < nível educacional \\
\hline \multirow[t]{2}{*}{ Dias et al. (2008a) } & $70 \mathrm{~TB}(\mathrm{eu})$ & SUMD & Insight (global e sobre tratamento) e idade & $<$ Insight: > idade \\
\hline & & & Insight sobre tratamento e nível educacional & $<$ Insight: < nível educacional \\
\hline Yen et al. (2004) & $65 \mathrm{~TB}(\mathrm{eu})$ & SAI-E e SAI-E & Insight e gênero & $<$ Insight: sexo masculino \\
\hline
\end{tabular}

TB: transtorno bipolar; mn: mania; ms: episódio misto; dp: depressão; eu: eutímico; SMS: Scale for Manic States; SUMD: Scale of Unawareness of Mental Disorders; SAl: Schedule for Assessment of Insight; SAI-E: Schedule for Assessment of Insight.

Tabela 3. Dados clínicos retrospectivos

\begin{tabular}{|c|c|c|c|c|}
\hline Estudo & Amostra & $\begin{array}{l}\text { Instrumento de } \\
\text { avaliação do insight }\end{array}$ & Variáveis & Resultados \\
\hline Acosta et al. (2012) & 102 TB (eu) & SUMD & Insight sobre a doença e história de ideação suicida & $\begin{array}{l}<\text { Insight: < história de ideação } \\
\text { suicida }\end{array}$ \\
\hline \multirow[t]{3}{*}{ Bressi et al. (2012) } & 120 TB (40 mn; $32 \mathrm{~ms} ; 48 \mathrm{dp})$ & SUMD & $\begin{array}{l}\text { Insight (sobre a doença e sobre tratamento) e } \\
\text { hospitalizações prévias }\end{array}$ & $\begin{array}{l}<\text { Insight sobre a doença: < número } \\
\text { hospitalizaçōes prévias } \\
<\text { Insight sobre tratamento: > } \\
\text { número hospitalizações prévias }\end{array}$ \\
\hline & & & Insight (sobre tratamento) e duração da doença & < Insight: > duração da doença \\
\hline & & & $\begin{array}{l}\text { Insight sobre consequências sociais e idade de } \\
\text { abertura }\end{array}$ & $<$ Insight: $<$ idade de abertura \\
\hline Güçlü et al. (2011) & $104 \mathrm{~TB}$ (mn ou ms) & SUMD & $\begin{array}{l}\text { Insight e número de hospitalizações ou número de } \\
\text { episódios prévios ou tipo do primeiro episódio }\end{array}$ & Sem correlação \\
\hline \multirow{3}{*}{$\begin{array}{l}\text { Van der Werf-Eldering et } \\
\text { al. (2011) }\end{array}$} & 85 TB (48 dp; 37 eu) & MDIS & Insight sobre a doença e história de psicose & $<$ Insight: história de psicose \\
\hline & & & Insight sobre sintomas e história de psicose & $\begin{array}{l}<\text { Insight: ausência de história } \\
\text { psicose }\end{array}$ \\
\hline & & & Insight sobre tratamento e duração da doença & < Insight: < duração \\
\hline
\end{tabular}


Tabela 3. Dados clínicos retrospectivos

\begin{tabular}{|c|c|c|c|c|}
\hline Estudo & Amostra & $\begin{array}{l}\text { Instrumento de } \\
\text { avaliação do insight }\end{array}$ & Variáveis & Resultados \\
\hline Cassidy (2010) & 156 TB (86 mn, 29 ms; 14 dp, 27 eu) & Item 13 SMS & $\begin{array}{l}\text { Insight e duração da doença ou número de } \\
\text { hospitalizações ou idade do primeiro episódio }\end{array}$ & Sem correlação \\
\hline \multirow[t]{4}{*}{ Dias et al. (2008b) } & $50 \mathrm{~TB}(\mathrm{eu})$ & SUMD & Insight sobre a doença e número de internações & $<$ Insight: < número de internações \\
\hline & & & Insight sobre consequências sociais e idade de abertura & $<$ Insight: > idade de abertura \\
\hline & & & $\begin{array}{l}\text { Insight global e número de episódios maníacos ou } \\
\text { número de episódios depressivos }\end{array}$ & Sem correlação \\
\hline & & & Insight e história de psicose & Sem correlação \\
\hline \multirow[t]{2}{*}{ Dias et al. (2008a) } & $70 \mathrm{~TB}(\mathrm{eu})$ & SUMD & $\begin{array}{l}\text { Insight global e número de episódios maníacos ou } \\
\text { número de episódios depressivos }\end{array}$ & Sem correlação \\
\hline & & & Insight sobre doença e história de psicose & <Insight: psicose pretérita \\
\hline Gonzalez (2008) & $297 \mathrm{~TB}$ & $\begin{array}{l}\text { Questão realizada ao } \\
\text { participante sobre ter } \\
\text { uma doença mental }\end{array}$ & $\begin{array}{l}\text { Insight sobre a doença e história de ideação ou } \\
\text { tentativa de suicídio }\end{array}$ & $\begin{array}{l}<\text { Insight: }<\text { história de ideação ou } \\
\text { tentativa de suicídio }\end{array}$ \\
\hline Yen et al. (2008c) & $96 \mathrm{~TB}(\mathrm{eu})$ & SAI e SAI-E & Insight e história de ideação ou tentativa de suicídio & $\begin{array}{l}<\text { Insight: < história de ideação ou } \\
\text { tentativa de suicídio }\end{array}$ \\
\hline Colis et al. (2006) & 52 TB (18 mn; $16 \mathrm{~ms} ; 18 \mathrm{dp})$ & $\mathrm{BCIS}$ & Insight e episódio afetivo anterior & $\begin{array}{l}<\text { Insight: episódio anterior de } \\
\text { mania }\end{array}$ \\
\hline Yen et al. (2004) & $65 \mathrm{~TB}(\mathrm{eu})$ & SAI-E e SAI-E & Insight (global e sobre sintomas) e duração da doença & $<$ Insight: < duração \\
\hline \multirow[t]{2}{*}{ Pini et al. (2003) } & $\begin{array}{l}151 \text { TB com psicose ( } 92 \text { sem } \\
\text { comorbidade; } 35 \text { com TP; } 24 \text { com } \\
\text { TOC ou FS) }\end{array}$ & SUMD & $\begin{array}{l}\text { Insight sobre a doença referente a episódio afetivo } \\
\text { prévio e comorbidade }\end{array}$ & $\begin{array}{l}<\text { Insight: TB com TP ou TB sem } \\
\text { comorbidade }\end{array}$ \\
\hline & & & $\begin{array}{l}\text { Insight sobre tratamento referente a episódio afetivo } \\
\text { prévio e comorbidade }\end{array}$ & $<$ Insight: TB com TP \\
\hline Lam et al. (1997) & $40 \mathrm{~TB}(\mathrm{eu})$ & IQ & Insight e lida (coping) com pródromos de hipomania & $\begin{array}{l}<\text { Insight: pior lida (coping) com } \\
\text { pródromos de hipomania }\end{array}$ \\
\hline Ghaemi et al. (1996) & $16 \mathrm{~TB}(\mathrm{mn})$ & ITAQ & Insight e número de hospitalizações prévias & $\begin{array}{l}<\text { Insight: < número hospitalizações } \\
\text { prévias (associação fraca) }\end{array}$ \\
\hline
\end{tabular}

TB: transtorno bipolar; mn: mania; ms: episódio misto; dp: depressão; eu: eutímico; TP: transtorno de pânico; TOC: transtorno obsessivo-compulsivo; FS: fobia social; SUMD: Scale of Unawareness of Mental Disorders; SMS: Scale for Manic States; BCIS: Beck Cognitive Insight Scale; PANSS: Positive and Negative Syndrome Scale; HDS: Hamilton Depression Scale; ITAQ: Insight and Treatment Attitudes Questionnaire; YMRS: Young Mania Rating Scale; MADP: Manual for the Assessment and Documentation in Psychopathology; MDIS: Mood Disorder Insight Scale; IQ: Insight Questionnaire.

Os três estudos ${ }^{12,51,52}$ que investigaram a relação entre insight e história de ideação suicida observaram que um menor insight sobre a doença foi associado a uma história menos frequente de ideação suicida. E os dois estudos ${ }^{51,52}$ que investigaram a relação entre insight e história de tentativa de suicídio também relataram que um menor insight estava associado a uma história menos frequente de tentativa de suicídio. Cinco estudos 17,18,44,49,55 investigaram a relação entre insight e número de hospitalizações prévias. Um menor insight foi associado a um menor número de hospitalizações prévias em três desses estudos ${ }^{17,49,55}$. Entretanto, os dois outros estudos ${ }^{18,44}$ não encontraram correlação entre insight e número de hospitalizações prévias. Quatro estudos 44,46,49,50 investigaram a relação entre duração da doença e insight. Tanto uma menor ${ }^{46,50}$ quanto uma maior ${ }^{49}$ duração da doença foram associados a um menor insight. Um estudo ${ }^{44}$ não encontrou correlação entre insight e duração da doença.
Três estudos investigaram a relação entre insight e a ocorrência prévia de sintomas psicóticos ${ }^{17,46,50}$. Um deles ${ }^{50}$ relatou que menor insight sobre a doença estava associado a uma história de sintomas psicóticos. Contudo, esse mesmo estudo ${ }^{50}$ relatou que menor insight sobre sintomas estava associado à ausência de história de sintomas psicóticos. Outro estudo ${ }^{46}$ relatou que menor insight sobre sintomas psicóticos estaria associado à história de sintomas psicóticos. E um estudo ${ }^{17}$ não encontrou correlação entre insight e história de sintomas psicóticos.

\section{Dados clínicos prospectivos}

Os 292-5,13,17,18,25,29,30,36,40-42,47,49,50,53,56-66 estudos sobre a relação entre o insight no TB e dados psicopatológicos prospectivos são apresentados na tabela 4.

Entre eles, sete estudos 13,29,30,40,49,65,66 investigaram a evolução do nível de insight e a resolução do episódio afetivo agudo, e todos os sete estudos relataram aumento do nível do insight quando ocorria a melhora do episódio afetivo. 
Tabela 4. Dados clínicos prospectivos

\begin{tabular}{|c|c|c|c|c|}
\hline Estudo & Amostra & $\begin{array}{l}\text { Instrumento de } \\
\text { avaliação do insight }\end{array}$ & Variáveis & Resultados \\
\hline \multirow[t]{2}{*}{ Bressi et al. (2012) } & \multirow[t]{2}{*}{120 TB (40 mn; 32 ms; 48 dp) } & \multirow[t]{2}{*}{ SUMD } & $\begin{array}{l}\text { Insight e resolução do episódio maníaco ou do episódio } \\
\text { misto }\end{array}$ & $\begin{array}{l}<\text { Insight: pior evolução de episódio } \\
\text { maníaco ou misto }\end{array}$ \\
\hline & & & $\begin{array}{l}\text { Insight (sobre a doença e consequências sociais) e } \\
\text { gravidade do episódio maníaco (escores de YMRS) }\end{array}$ & $\begin{array}{l}<\text { Insight: > gravidade do episódio } \\
\text { maníaco }\end{array}$ \\
\hline Calatayud et al. (2012) & TB com psicose $(n=43)$ & PANSS G12 & Insight e hostilidade ou controle dos impulsos & $\begin{array}{l}<\text { Insight: }>\text { hostilidade e } \\
<\text { controle dos impulsos }\end{array}$ \\
\hline Cerit et al. (2012) & 80 TB (eu) & SAI & Insight e funcionamento psicossocial & Sem correlação \\
\hline Cely et al. (2011) & 124 TB sem psicose & $\begin{array}{l}\text { Item de questionário } \\
\text { criado pelos autores }\end{array}$ & Insight e adesão ao tratamento farmacológico & $<$ Insight: < adesão \\
\hline Güçlü et al. (2011) & 104 TB (mn ou ms) & SUMD & Insight sobre tratamento e presença de psicose & $<$ Insight: presença de psicose \\
\hline $\begin{array}{l}\text { Van der Werf-Eldering et } \\
\text { al. (2011) }\end{array}$ & 85 TB (48 dp; $37 \mathrm{eu})$ & MDIS & $\begin{array}{l}\text { Insight (global, sobre a doença e tratamento) e } \\
\text { número de sintomas depressivos }\end{array}$ & $\begin{array}{l}<\text { Insight: < número de sintomas } \\
\text { depressivos }\end{array}$ \\
\hline $\begin{array}{l}\text { González-Ortega et al. } \\
\text { (2010) }\end{array}$ & 173 TB (136 mn; 37 ms) & Item 11 da YMRS & Insight e comportamento agressivo & $\begin{array}{l}<\text { Insight: > comportamento } \\
\text { agressivo }\end{array}$ \\
\hline Sajatovic et al. (2009) & $140 \mathrm{~TB}$ & ITAQ & Insight sobre a doença e adesão ao tratamento & < Insight: < adesão \\
\hline \multirow[t]{2}{*}{ Varga et al. (2008) } & \multirow[t]{2}{*}{21 TB (1 mn; 7 dp; 13 eu) } & \multirow[t]{2}{*}{ SUMD } & Insight e CT & $\begin{array}{l}<\text { Insight global: atrofia subcortical; < } \\
\text { insight sobre sintomas: atrofia cortical }\end{array}$ \\
\hline & & & Insight e SPECT & Sem correlação \\
\hline Copeland et al. (2008) & $435 \mathrm{~TB}$ & $\begin{array}{l}\text { Escalas adaptadas de } \\
\text { Meredith et al. (2002) }\end{array}$ & Insight e adesão ao tratamento farmacológico & $<$ Insight: < adesão \\
\hline \multirow[t]{2}{*}{ Dias et al. (2008b) } & \multirow[t]{2}{*}{$50 \mathrm{~TB}(\mathrm{eu})$} & \multirow[t]{2}{*}{ SUMD } & Insight sobre a doença e número de sintomas maníacos & $<$ Insight: $>$ número de sintomas \\
\hline & & & Insight e presença de sintomas depressivos & Sem correlação \\
\hline \multirow[t]{2}{*}{ Dias et al. (2008a) } & \multirow[t]{2}{*}{$70 \mathrm{~TB}(\mathrm{eu})$} & \multirow[t]{2}{*}{ SUMD } & Insight sobre doença e sintomas maníacos & $\begin{array}{l}<\text { Insight: > número sintomas } \\
\text { maníacos }\end{array}$ \\
\hline & & & Insight global e qualidade de vida autorrelatada & $\begin{array}{l}<\text { Insight: < qualidade de vida } \\
\text { ambiental e psicológica }\end{array}$ \\
\hline \multirow[t]{2}{*}{ Yen et al. (2008b) } & \multirow[t]{2}{*}{$65 \mathrm{~TB}(\mathrm{eu})$} & \multirow[t]{2}{*}{ SAI } & $\begin{array}{l}\text { Insight sobre tratamento e maior gravidade do } \\
\text { episódio }\end{array}$ & $\begin{array}{l}<\text { Insight: maior gravidade do } \\
\text { episódio }\end{array}$ \\
\hline & & & $\begin{array}{l}\text { Insight (sobre a doença e sobre sintomas psicóticos) e } \\
\text { maior gravidade do episódio }\end{array}$ & Sem correlação \\
\hline Yen et al. (2008d) & $\begin{array}{l}19 \text { TB (comorbidade uso de álcool) } \\
\text { e } 40 \text { TB (sem comorbidade uso } \\
\text { de álcool) }\end{array}$ & SAI-E & Insight e uso de álcool em TB & Sem correlação \\
\hline Yen et al. (2008e) & $96 \mathrm{~TB}(\mathrm{eu})$ & SAI-E & Insight e qualidade de vida & $\begin{array}{l}<\text { Insight: > qualidade de vida no } \\
\text { domínio físico }\end{array}$ \\
\hline Yen et al. (2007b) & $50 \mathrm{~TB}(\mathrm{eu})$ & SAl e SAI-E & Insight e ajustamento social & $<$ Insight: < ajustamento social \\
\hline Varga et al. (2007) & $\begin{array}{l}37 \text { TB ( } 5 \text { mn; } 10 \text { dp; } 17 \text { em remissão } \\
\text { total; } 5 \text { em remissão parcial) }\end{array}$ & SUMD & Insight global e gravidade clínica & $<$ Insight: > gravidade \\
\hline Schuepbach et al. (2006) & $95 \mathrm{~TB}$ (mn ou ms) & Item: falta de insight & Insight e admissão internação involuntária & $\begin{array}{l}<\text { Insight: admissão hospitalar } \\
\text { involuntária }\end{array}$ \\
\hline Varga et al. (2006) & $37 \mathrm{~TB}$ & SUMD & $\begin{array}{l}\text { Insight (sobre doença e sobre sintomas) e gravidade } \\
\text { da doença }\end{array}$ & $<$ Insight: > gravidade \\
\hline Yen et al. (2005) & 60 TB (9 mn; 5 dp; 46 eu) & SAl e SAI-E & $\begin{array}{l}\text { Insight (global, sobre a doença, sobre tratamento e } \\
\text { sintomas psicóticos) e adesão medicamentosa }\end{array}$ & < Insight: < adesão \\
\hline \multirow[t]{2}{*}{ Pini et al. (2003) } & \multirow[t]{2}{*}{$\begin{array}{l}151 \text { TB com psicose ( } 92 \text { sem } \\
\text { comorbidade; } 35 \text { com TP; } 24 \text { com } \\
\text { TO( ou FS) }\end{array}$} & \multirow[t]{2}{*}{ SUMD } & $\begin{array}{l}\text { Insight atual (sobre a doença e tratamento) e } \\
\text { comorbidade }\end{array}$ & $\begin{array}{l}<\text { Insight: TB com TP ou TB sem } \\
\text { comorbidade }\end{array}$ \\
\hline & & & Insight e TP & < Insight: presença de TP \\
\hline \multirow[t]{2}{*}{ Yen et al. (2003) } & \multirow[t]{2}{*}{33 TB (mn) } & \multirow[t]{2}{*}{ SAI-E e SAI } & Insight e resolução do episódio maníaco & < Insight: pior evolução do episódio \\
\hline & & & Insight sobre tratamento e psicose & $<$ Insight: presença de psicose \\
\hline Dell'0sso et al. (2002) & $\begin{array}{l}147 \text { TB com psicose ( } 55 \mathrm{mn} ; 62 \\
\mathrm{~ms} ; 30 \mathrm{dp})\end{array}$ & SUMD & Insight e sintomas maníacos específicos & Sem correlação \\
\hline
\end{tabular}


Tabela 4. Dados clínicos prospectivos

\begin{tabular}{|c|c|c|c|c|}
\hline Estudo & Amostra & $\begin{array}{l}\text { Instrumento de } \\
\text { avaliação do insight }\end{array}$ & Variáveis & Resultados \\
\hline Ghaemi et al. (2000) & $45 \mathrm{~TB}$ & SUMD & Melhora de insight e evolução do tratamento & $<$ Melhora de insight: pior evolução \\
\hline Weiler et al. (2000) & $40 \mathrm{~TB}$ & ITAQ & Insight global e resolução da crise & < Insight: pior evolução da crise \\
\hline Peralta e Cuesta (1998) & 29 TB (21 mn; $7 \mathrm{dp})$ & MADP & Insight e resolução da crise & < Insight: pior evolução da crise \\
\hline Fennig et al. (1996) & $52 \mathrm{~TB}$ & $\begin{array}{l}\text { Item modificado de } \\
\text { HDS }\end{array}$ & Insight e resolução da crise & < Insight: pior evolução da crise \\
\hline \multirow[t]{2}{*}{ Ghaemi et al. (1995) } & $28 \mathrm{~TB}(\mathrm{mn})$ & ITAQ & Insight e resolução da crise & < Insight: pior evolução da crise \\
\hline & & & Insight e hospitalização involuntária & $\begin{array}{l}\text { < Insight: hospitalização } \\
\text { involuntária }\end{array}$ \\
\hline Michalakeas et al. (1994) & $13 \mathrm{~TB}(\mathrm{mn})$ & ITAQ & Insight global e resolução da crise & < Insight: pior evolução da crise \\
\hline
\end{tabular}

TB: transtorno bipolar; mn: mania; ms: episódio misto; dp: depressão; eu: eutímico; TP: transtorno de pânico; TOC: transtorno obsessivo-compulsivo; FS: fobia social; SUMD: Scale of Unawareness of Mental Disorders: SMS: Scale for Manic States; PANSS: Positive and Negative Syndrome Scale; HDS: Hamilton Depression Scale; ITAQ: Insight and Treatment Attitudes Questionnaire; YMRS: Young Mania Rating Scale; MADP: Manual for the Assessment and Documentation in Psychopathology; MDIS: Mood Disorder Insight Scale; CT: computed tomography; SPECT: single-photon emission computed tomography.

Os quatro estudos ${ }^{36,49,59,64}$ que avaliaram a correlação entre insight e gravidade do episódio afetivo encontraram correlação entre menor nível de insight e maior gravidade do episódio afetivo. Os dois estudos ${ }^{18,65}$ que avaliaram a relação entre presença de sintomas psicóticos e insight observaram que menor insight estava associado à presença de sintomas psicóticos. Os quatro estudos ${ }^{2,3,4,5}$ que avaliaram a relação entre insight e adesão ao tratamento farmacológico observaram que menor insight estava associado a menor adesão ao tratamento. Os dois estudos ${ }^{13,63}$ que investigaram a relação entre insight e hospitalização involuntária observaram que menor nível de insight estava associado à hospitalização involuntária. Alguns estudos isolados relataram que menor insight estava também associado a maior hostilidade e menor controle dos impulsos ${ }^{25}$, maior comportamento agressivo ${ }^{57} \mathrm{e}$ pior evolução clínica ${ }^{41}$. E, ainda, um estudo ${ }^{58} \mathrm{com}$ tomografia computadorizada encontrou que menor insight estava associado à atrofia subcortical e cortical.

\section{Avaliação neuropsicológica}

Os nove estudos $17,36,38,45,47,50,55,64,67$ sobre a relação entre o insight no TB e avaliações neuropsicológicas são apresentados na tabela 5.

Foram encontradas associações entre um menor insight e um maior comprometimento de memória ${ }^{17,45,47,50}$ e de aprendizagem emocional50, de inteligência geral e de função psicomotora ${ }^{36,64}$, de compreensão e de habilidades perceptivo-motoras ${ }^{17,47}$, de atenção ${ }^{17,36,64}$, de fluência verbal ${ }^{17,67}$ e de função executiva ${ }^{17,45,47}$, e ainda de maior velocidade de processamento ${ }^{50}$

\section{Comparação entre os diversos estados afetivos do TB}

Os nove estudos ${ }^{22,42,44,49,66,68-71}$ sobre a comparação quanto ao insight entre os diversos estados afetivos do TB são apresentados na tabela 6.
Todos os seis estudos ${ }^{42,44,49,66,68,71}$ que compararam maníacos e deprimidos bipolares relataram que o insight de maníacos era menor do que o de deprimidos bipolares. Sete estudos $22,42,44,49,69-71$ compararam pacientes em mania com pacientes em episódio misto. Desses, cinco estudos ${ }^{22,42,44,69,71}$ relataram que o insight de maníacos era menor do que o de pacientes em episódio misto; em contraste, dois estudos ${ }^{12,70}$ relataram que $\mathrm{o}$ insight em pacientes em mania é maior do que o de pacientes em episódio misto. Os dois estudos ${ }^{44,68}$ que compararam o insight entre maníacos e eutímicos relataram que o insight dos maníacos era menor do que o dos eutímicos. Quatro estudos ${ }^{42,44,49,71} \mathrm{com}$ pararam pacientes deprimidos e em episódio misto. Desses estudos, apenas um estudo ${ }^{12}$ relatou que insight de pacientes em episódio misto era menor do que de deprimidos. Os outros três ${ }^{42,44,71}$ não encontraram diferenças significativas. Por fim, apenas um estudo ${ }^{68}$ comparou eutímicos e deprimidos e relatou que insight dos eutímicos era igual ao dos deprimidos.

\section{DISCUSSÃO}

O insight é a capacidade do indivíduo de avaliar criticamente seu estado mental ${ }^{72}$. O presente estudo realizou uma revisão sistemática dos trabalhos que investigaram o insight em pacientes com TB. É importante salientar que o comprometimento do insight parece ser um fenômeno bastante abrangente, sendo encontrado em diversos transtornos mentais, como a esquizofrenia ${ }^{7-10}$, e doenças neurológicas. Estudos sobre doença de Alzheimer comumente empregam o ter-

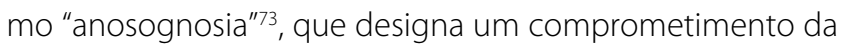
capacidade de perceber em si ou nas atividades da vida diária alterações causadas por déficits relacionados ao processo de adoecimento ${ }^{74}$. Esse fenômeno é comumente associado a inúmeras alterações presentes na doença de Alzheimer ${ }^{73}$. 
Tabela 5. Avaliação neuropsicológica

\begin{tabular}{|c|c|c|c|c|}
\hline Estudo & Amostra & $\begin{array}{l}\text { Instrumento de } \\
\text { avaliação do insight }\end{array}$ & Avaliação neuropsicológica & Resultados \\
\hline $\begin{array}{l}\text { Van der Werf- } \\
\text { Eldering et al. (2011) }\end{array}$ & $\begin{array}{l}85 \mathrm{~TB}(48 \mathrm{dp} ; \\
37 \mathrm{eu})\end{array}$ & MDIS & CANTAB (RTI, PRM e SWM), SCWT, CVLT, CPT, IQ, ELT & $\begin{array}{l}<\text { Insight global: < memória; e melhor velocidade } \\
\text { de processamento } \\
<\text { Insight sobre a doença: < memória; } \\
<\text { aprendizado emocional; e melhor velocidade de } \\
\text { processamento }\end{array}$ \\
\hline Dias et al. (2008b) & $50 \mathrm{~TB}(\mathrm{eu})$ & SUMD & $\begin{array}{l}\text { WMS (MT, DS, LM), SDMT, TMT-A, SCT, SC-WT, } \\
\text { TMT-B, ToH, COWAT, WAIS-R (comprehension, } \\
\text { similarities and information sub-tests), BT }\end{array}$ & $\begin{array}{l}<\text { Insight global: < performance em comprehension, } \\
\text { similarities and information sub-tests, MT, DS, } \\
\text { SDMT, TMT-A, TMT-B, SCT (perseverations), ToH, } \\
\text { and COWAT }\end{array}$ \\
\hline Dias et al. (2008a) & $70 \mathrm{~TB}(\mathrm{eu})$ & SUMD & $\begin{array}{l}\text { WMS (MT, DS, LM), SDMT, TMT-A, SCT, SCWT, } \\
\text { TMT-B, ToH, COWAT, WAIS-R (comprehension, } \\
\text { similarities and information sub-tests), BT }\end{array}$ & $\begin{array}{l}<\text { Insight global: < performance em SDMT, TMT-A, } \\
\text { TMT-B, SCT (perseverations), ToH, Similarities sub- } \\
\text { tests of the WAIS-R, and in LM }\end{array}$ \\
\hline Yen et al. (2008a) & $96 \mathrm{~TB}(\mathrm{eu})$ & SAI E SAl-E & WCST, WAIS-III, WMS-III, CPT, COWAT, LCT & $\begin{array}{l}<\text { Insight (global, sobre a doença, e } \\
\text { reconhecimento de fenômeno): função executiva e } \\
\text { memória prejudicados }\end{array}$ \\
\hline Varga et al. (2007) & $\begin{array}{l}37 \text { TB }(5 \mathrm{mn} ; \\
10 \mathrm{dp} ; 17 \text { em } \\
\text { remissão total; } \\
5 \text { em remissão } \\
\text { parcial) }\end{array}$ & SUMD & $\begin{array}{l}\text { WCST, SCWT, TMT-A, TMT-B, WAIS-III (DS, DSy, BD, } \\
\text { information and similarities), AVLT, IL, GP }\end{array}$ & $\begin{array}{l}<\text { Insight global: < performance em WAIS } \\
\text { (similarities), GP dominant hand e WAIS (DS) }\end{array}$ \\
\hline Varga et al. (2006) & $37 \mathrm{~TB}$ & SUMD & $\begin{array}{l}\text { WCST, SCWT, TMT-A, TMT-B, WAIS-III (DS, DSy, BD, } \\
\text { information and similarities), AVLT, IL, GP }\end{array}$ & $\begin{array}{l}<\text { Insight global: < performance em WAIS } \\
\text { (similarities), GP dominant hand e WAIS (DS) }\end{array}$ \\
\hline Arduini et al. (2003) & $\begin{array}{l}22 \mathrm{~TB}(\mathrm{mn} \text { com } \\
\text { psicose) }\end{array}$ & SUMD & WCST & Sem correlação \\
\hline Yen et al. (2002a) & $34 \mathrm{~TB}(\mathrm{eu})$ & SAI-E & WCST, WAIS-R, WMS, TMT, CPT, COWAT, VFDT, LCT & < Insight: < performance COWAT (associação fraca) \\
\hline Ghaemi et al. (1996) & $16 \mathrm{~TB} \mathrm{mn}$ & ITAQ & $\begin{array}{l}\text { WAIS-R, WMS, COWAT, FTT, screening de } \\
\text { linguagem, testes de habilidades construtivas } \\
\text { visuais e perceptivas visuais, medidas de funções } \\
\text { motoras de autocontrole }\end{array}$ & $\begin{array}{l}<\text { Insight: < anormalidades da memória visual } \\
\text { retardada (associação fraca) }\end{array}$ \\
\hline
\end{tabular}

TB: transtorno bipolar; mn: mania; dp: depressão; eu: eutímico; SUMD: Scale of Unawareness of Mental Disorders; ITAQ: Insight and Treatment Attitudes Questionnaire; MDIS: Mood Disorder Insight Scale; SAI: Schedule for Assessment of Insight; SAI-E: Schedule for Assessment of Insight; AVLT: Auditory Verbal Learning Test; BD: Block Design; BNLN: Neuropsychological Battery Luria-Nebraska; BT: Bell's Test; CANTAB: Cambridge Neuropsychological Test Automated Battery; COWAT: Controlled Oral Word Association Test; CPT: Continuous Performance Task; CVLT: California Verbal Learning Test; DS: Digit Span; DSy: Digit Symbol; ELT: Emotional Learning Task; FTT: Finger Tapping Test; GP: Grooved Pegboard; IL: Incidental Learning; IQ: Intelligence Quotient; LCT: Line Cancellation Test; LM: Logical Memory; MT: Mental Tracking; PRM: Pattern Recognition Memory; RTI: Reaction Time Test; SCT: Stroop Colour Test; SC-WT: Stroop Colour-Write Test; SCWT: Stroop Colour and Word Test; SDMT: Symbol Digit Modalities Test; SWM: Spatial Working Memory; TMT: Trail Making Test; TMT-A: Trail Making Test part A; TMT-B: Trail Making Test part B; ToH: Hanoi Towers Test; VFDT: Visual Form Discrimination Test; WAIS-III: Wechsler Adult Intelligence Scale - third edition; WAIS-R: Wechsler Adult Intelligence Scale-Revised; WCST: Wisconsin Card Sorting Test; WMS: Wechsler Memory Scale; WMS-III: Wechsler Memory Scale - third edition.

Tabela 6. Comparação entre os diversos estados afetivos do transtorno bipolar

\begin{tabular}{|c|c|c|c|c|}
\hline Estudo & Amostra e desenho & Tipo de insight & Instrumento de avaliação & Resultados \\
\hline \multirow[t]{3}{*}{ Bressi et al. (2012) } & 120 TB: 40 mn vs. 32 ms vs. $48 \mathrm{dp}$ & Sobre a doença & SUMD & $\mathrm{mn}<\mathrm{dp} ; \mathrm{ms}<\mathrm{dp}$ \\
\hline & & Sobre tratamento & & $\mathrm{ms}<\mathrm{dp}$ \\
\hline & & Sobre consequências sociais & & $\mathrm{ms}<\mathrm{mn}<\mathrm{dp}$ \\
\hline Cassidy (2010) & $156 \mathrm{~TB}: 86 \mathrm{mn}$ vs. $29 \mathrm{~ms}$ vs. $14 \mathrm{dp}$ vs. $27 \mathrm{eu}$ & Global & item 13 SMS & $\mathrm{mn}<\mathrm{dp} ; \mathrm{mn}<\mathrm{ms} ; \mathrm{mn}<\mathrm{eu}$ \\
\hline Yen et al. (2007a) & $\begin{array}{l}65 \text { TB acompanhados por } 2 \text { anos (16: um } \\
\text { episódio } \mathrm{mn} ; 3 \text { : um episódio dp; } 6 \text { mais de um } \\
\text { episódio } \mathrm{mn} \text { ou dp) }\end{array}$ & Global & SAI-E & $\mathrm{mn}<\mathrm{eu}=\mathrm{dp}$ \\
\hline Pini et al. (2004) & 78 TB com psicose: $29 \mathrm{mn}$ vs. $49 \mathrm{~ms}$ & Sobre consequências sociais & SUMD & $\mathrm{mn}<\mathrm{ms}$ \\
\hline \multirow[t]{3}{*}{ Dell'0sso et al. (2002) } & 147 TB com psicose: 55 mn vs. 62 ms vs. $30 \mathrm{dp}$ & Sobre a doença & SUMD & $\mathrm{mn}<\mathrm{dp}$ \\
\hline & & Sobre tratamento & & $\mathrm{mn}<\mathrm{ms} ; \mathrm{mn}<\mathrm{dp}$ \\
\hline & & Sobre consequências sociais & & $\mathrm{mn}<\mathrm{ms} ; \mathrm{mn}<\mathrm{dp}$ \\
\hline Cassidy et al. (2001) & 53 TB: $42 \mathrm{mn}$ vs. $11 \mathrm{~ms}$ & Global & ITAQ & $\mathrm{mn}<\mathrm{ms}$ \\
\hline Rossi et al. (2000) & $124 \mathrm{mn}$ vs. $22 \mathrm{~ms}$ & Global & PANSS G12 & $\mathrm{ms}<\mathrm{mn}$ \\
\hline Dell'Osso et al. (2000) & 125 TB psicóticos: 62 mn vs. 28 ms vs. 35 dp & Sobre consequências sociais & SUMD & $\mathrm{mn}<\mathrm{dp} ; \mathrm{mn}<\mathrm{ms}$ \\
\hline Peralta e Cuesta (1998) & 28 TB: $21 \mathrm{mn}$ vs. $7 \mathrm{dp}$ & Global & MADP & $\mathrm{mn}<\mathrm{dp}$ \\
\hline
\end{tabular}

TB: transtorno bipolar; mn: mania; ms: episódio misto; dp: depressão; eu: eutímico; SUMD: Scale of Unawareness of Mental Disorders; SMS: Scale for Manic States; BCIS: Beck Cognitive Insight Scale; PANSS: Positive and Negative Syndrome Scale; HDS: Hamilton Depression Scale; ITAQ: Insight and Treatment Attitudes Questionnaire; YMRS: Young Mania Rating Scale; MADP: Manual for the Assessment and Documentation in Psychopathology; MDIS: Mood Disorder Insight Scale; IQ: Insight Questionnaire; <: menor insight que; >: maior insight que; = : sem diferenças quanto ao insight. 
Além disso, grande parte dos pacientes com transtorno obsessivo-compulsivo (TOC) é parcial ou totalmente incapaz de reconhecer a irracionalidade de seus sintomas, ou seja, apresenta pouco ou nenhum insight ${ }^{75}$.

Dentre as associações estudadas entre o insight no TB e dados sociodemográficos, algumas merecem ser destacadas. Alguns estudos ${ }^{17,45,47}$ mostram que maior nível educacional está relacionado a maior nível de insight. Provavelmente os pacientes com maior nível educacional tiveram maior acesso ao conhecimento sobre a sua doença e sobre as formas de lidar com ela ${ }^{17}$. É possível também que eles possuam maior status socioeconômico, com acesso a melhores serviços de saúde e formas de tratamento. Estudos em pacientes com demência também ressaltam a importância da escolaridade sobre o insight, pois melhor capacidade cognitiva pré-mórbida está relacionada a maior reserva cognitiva e, consequentemente, a menor comprometimento da consciência do déficit ${ }^{76}$. Entretanto, o baixo número de estudos sobre nível educacional não permite concluir que, de fato, o tempo de estudo de um paciente possa ser determinante para melhor insight.

Três estudos ${ }^{46,49,50}$ observaram que uma maior duração ou idade de início da doença mais precoce estariam associadas a menor insight no TB. Esses dados também poderiam reforçar a ideia de que um maior período de convivência com a doença levaria a melhor conhecimento sobre sua sintomatologia. Estudos com pacientes com $\mathrm{TOC}^{77}$ indicam que esses fatores estariam relacionados ao insight também nesse transtorno mental. Porém, em virtude do baixo número de estudos sobre o tema, a relação do nível de insight com esses fatores ainda precisa ser confirmada.

Outra constatação que parece evidente é a de que, no $\mathrm{TB}$, um comprometimento cognitivo pode estar relacionado a um comprometimento do insight. Níveis reduzidos de insight foram associados com piores desempenhos em testes de funções executivas ${ }^{17,47}$, atenção dividida, flexibilidade mental, memória de trabalho, inibição de resposta, resolução de interferência e resolução de conflito de comportamento, todos mediados pelo lobo frontal. Além disso, um prejuízo na fluência verbal foi significativamente associado com comprometimento do insight ${ }^{17}$. Esses resultados reforçam a noção de que o insight é uma capacidade neurocognitiva, com os achados indicando especificamente disfunção pré-frontal e frontoparietal em pacientes bipolares com baixo insight $t^{17,64}$. Os lobos frontais estão envolvidos em habilidades metacognitivas e de autoavaliação, tendo sido implicados na deficiência de insight em diferentes grupos clínicos, como pacientes com doença de Alzheimer ${ }^{19}$. Alguns estudos $17,36,45,50,64$ apontaram que um maior comprometimento de memória tem um impacto negativo sobre o nível de insight. Capacidade limitada de reter informação na memória verbal ou de se lembrar de comportamentos prévios pode ter impacto negativo na habilidade dos pacientes de avaliarem seu próprio comportamento e de tirarem conclusões sobre a anormalidade de seu comportamento ${ }^{50}$. Esses achados estão de acordo com uma extensa literatura que indica baixos níveis de insight em pacientes com comprometimentos de memória, por exemplo, na demência ${ }^{73}$. Pacientes com TOC e pouco insight também apresentam desempenho cognitivo prejudicado ${ }^{78}$.

Os estudos parecem ser unânimes ao apontar que, no TB, uma melhora da sintomatologia da crise cursa com recuperação do insight ${ }^{13,29,30,40,49,53,66}$. Observação semelhante foi relatada em um estudo com esquizofrênicos ${ }^{10}$, no qual ocorreu aumento do insight durante o período entre a hospitalização e a alta. Nesse mesmo sentido, estudos com pacientes com $\mathrm{TB}^{54} \mathrm{e}$ com esquizofrenia ${ }^{10}$ mostram que os pacientes, quando estão num período prodrômico, em que os sintomas estão menos intensos, são mais capazes de se queixar das alterações que estão apresentando, o que não acontece quando os sintomas se tornam mais graves. Esses dados apontam que a presença de sintomatologia aguda interfere no nível de discernimento do indivíduo. De fato, dados coletados de estudos, pela presente revisão, mostram uma relação direta entre menor insight no TB e uma gravidade do episódio afetivo ${ }^{36,49,59,64}$ ou da quantidade de sintomas maníacos ${ }^{17,30,32,47}$. De forma semelhante, pacientes com doença de Alzheimer apresentam piora da consciência do déficit com a progressão da doença e consequente aumento da gravidade clínica ${ }^{79,80}$. Maior comprometimento do insight associado a maior gravidade da doença também pode ser observado no TOC ${ }^{81}$. Contudo, no TB, a associação entre baixo nível de insight e gravidade clínica poderia ser explicada pelo fato de, nos episódios afetivos mais graves, serem especialmente comuns os sintomas psicóticos, os quais costumam cursar com importante prejuízo no insight ${ }^{18,46,50,65}$.

Entre os estudos que fizeram comparação do insight de pacientes bipolares com pacientes com outros transtornos mentais, 17 utilizaram pacientes esquizofrênicos. Nenhum estudo encontrou que o insight de esquizofrênicos fosse maior do que de pacientes bipolares. Contudo, alguns estudos encontraram níveis de insight semelhantes na comparação entre esquizofrênicos e bipolares ${ }^{11,22,23,31,36-39}$. É importante ressaltar que alguns desses estudos que não distinguiram TB de esquizofrenia utilizaram uma amostra de bipolares com sintomas psicóticos ${ }^{11,22,38}$. Além disso, conforme discutido, a presença de sintomas psicóticos parece ter grande influência sobre o insight ${ }^{18,65}$. A maioria dos estudos, contudo, indica que a esquizofrenia cursa com pior insight do que o TB. É possível que maior presença de sintomas psicóticos, maior comprometimento cognitivo e pior evolução da esquizofrenia ${ }^{82}$ possam ser responsáveis por um pior comprometimento do insight nesse transtorno. Um achado comum entre os estudos é que pacientes deprimidos unipolares apresentam melhor insight do que pacientes bipolares $23,29,30,40$. Um estudo ${ }^{43}$ chamou atenção para o fato de que pacientes bipolares tipo I apresentaram maior insight global do que pacientes bipolares tipo II, o que parece refletir a dificuldade, do próprio paciente e das demais pessoas, de fazer distinção entre a hipomania e a normalidade. 
Os estudos sobre comparação de insight nas diversas fases afetivas parecem ser unânimes ao apontar que pacientes em fase maníaca apresentam insight mais comprometido do que pacientes em fase depressiva ${ }^{42,44,49,66,68,71}$ ou em eutimia ${ }^{44,68}$. Além disso, um estudo mostrou que o insight de pacientes em fase depressiva é semelhante ao de pacientes em eutimia ${ }^{68}$. A associação entre humor deprimido e insight preservado é observada em diversos grupos clínicos ${ }^{27}$, mas a direção de causalidade não é clara nesse caso. Por um lado, um aumento do insight leva ao reconhecimento de problemas, o que causa tristeza. Por outro, pacientes deprimidos podem ser mais realistas, tendo maior apreciação das consequências de seus problemas. Nesse sentido, pacientes com TB que apresentam um episódio depressivo avaliam de forma mais fidedigna o seu estado de humor do que aqueles que apresentam um episódio maníaco ${ }^{14,83}$. Alguns estudos ${ }^{22,42,44,69,71}$ chamaram a atenção para o fato de que pacientes em mania apresentaram pior insight do que o de pacientes em episódio misto. Talvez a presença de sintomas depressivos possa favorecer um menor comprometimento do insight ${ }^{50}$. Três estudos ${ }^{48,51,52}$ encontraram que maior insight está associado a história de ideação ou tentativa de suicídio. Tais achados sugerem que a presença de insight sobre a doença pode ser um fator de risco para suicídio, o que pode estar relacionado à maior ocorrência de sintomas depressivos entre pacientes suicidas. Outra possibilidade seria que ter um nível maior de insight representa saber mais sobre a gravidade e consequências de sua doença e que a morte seria vista pelo indivíduo como uma alternativa menos dolorosa em relação ao TB.

A presença de apenas um juiz para o julgamento dos artigos a serem selecionados configura uma limitação da presente revisão sistemática.

\section{CONCLUSÃO}

Um menor nível de insight está relacionado à presença de sintomas psicóticos e de alterações cognitivas. Um comprometimento do insight está associado a uma menor adesão ao tratamento. Por outro lado, maior preservação do insight pode estar associada a maior ideação suicida. Finalmente, o insight no TB parece ser mais prejudicado do que na depressão unipolar, porém menos do que na esquizofrenia. E também está claro que a fase maníaca cursa com um nível inferior de insight quando comparada à fase depressiva ou de eutimia.

\section{CONTRIBUIÇÕES INDIVIDUAIS}

Rafael de Assis da Silva - Participou do desenho do estudo, da coleta e análise dos artigos selecionados e da redação do manuscrito e aprovou a versão final a ser publicada.
Daniel C. Mograbi - Participou do desenho do estudo e da redação do manuscrito e aprovou a versão final a ser publicada.

J. Landeira-Fernandez - Participou do desenho do estudo e da redação do manuscrito e aprovou a versão final a ser publicada.

Elie Cheniaux - Participou do desenho do estudo, da análise dos artigos selecionados e da redação do manuscrito e aprovou a versão final a ser publicada.

\section{CONFLITOS DE INTERESSE}

Os autores declaram não haver conflitos de interesse.

\section{REFERÊNCIAS}

1. Lewis AJ. The psychopathology of insight. Br J Med Psychol. 1934;14:332-48.

2. Cely EEP, Fierro M, Pinilla MI. Prevalencia y factores asociados a la no adherencia en el tratamento farmacológico de mantenimiento em adultos com transtorno afectivo bipolar. Rev Colomb Psiquiatr. 2011;40(1):85-98.

3. Copeland LA, Zeber JE, Salloum IM, Pincus HA, Fine MJ, Kilbourne AM. Treatment adherence and illness insight in veterans with bipolar disorder. J Nerv Ment Dis. 2008;196(1): $16-21$.

4. Sajatovic M, Ignacio RV, West JA, Cassidy KA, Safavi R, Kilbourne AM, et al. Predictors of nonadherence among individuals with bipolar disorder receiving treatment in a community mental health clinic. Comprehensive Psychiatry. 2009;50(2):100-7.

5. Yen CF, Chen CS, Ko CH, Yeh ML, Yang SJ, Yen JY, et al. Relationships between insight and medication adherence in outpatients with schizophrenia and bipolar disorder: prospective study. Psychiatry Clin Neurosci. 2005;59(4):403-9.

6. Amador XF, Flaum M, Andreasen NC, Strauss DH, Yale SA, Clark SC, et al. Awareness of illness in schizophrenia and schizoaffective and mood disorders. Arch Gen Psychiatry. 1994:51(10):826-36.

7. McEvoy JP, Apperson LJ, Appelbaum PS, Ortlip P, Brecosky J, Hammill K. Insight in schizophrenia. Its relationship to acute psychopathology. J Nerv Ment Dis. 1989;177(1):43-7.

8. Parellada M, Boada L, Fraguas D, Reig S, Castro-Fornieles J, Moreno D, et al. Trait and state attributes of insight in first episodes of early-onsent schizophrenia and other psychoses: a 2-year longitudinal study. Schizophr Bull. 2011;37(1):38-51.

9. Antonius D. Insight and aggression in schizophrenia. Am J Psychiatry. 2005;162(11):2195.

10. Dam J. Insight in schizophrenia: a review. Nord J Psychiatry. 2006;60(2):114-20.

11. Pini S, Cassano GB, Dell'Osso L, Amador XF. Insight into illness in schizophrenia, schizoaffective and mood disorders with psychotic features. Am J Psychiatry. 2001;158(1):122-5.

12. Ghaemi SN, Sachs GS, Baldassano (F, Truman CJ. Insight in seasonal affective disorder. Compr Psychiatry. 1997;38(6):345-8.

13. Ghaemi SN, Stoll AL, Pope HG. Lack of insight in bipolar disorder: the acute manic episode. J Nerv Ment Dis. 1995;183(7):464-7.

14. Silva RA, Mograbi DC, Silveira LAS, Nunes ALS, Novis FD, Cavaco PA, et al. Mood selfassessment in bipolar disorder: a comparison between patients in mania, depression, and euthymia. Trends Psychiatry Psychoter. 2013;35(2):141-5.

15. Ghaemi SN, Boiman EE, Goodwin FK. Diagnosing bipolar disorder and the effect of antidepressants: a naturalistic study. J Clin Psychiatry. 2000a;61(10):804-8.

16. Ghaemi SN, Ko JY, Goodwin FK. "Cade's disease" and beyond: misdiagnosis, antidepressant use and a proposed definition for bipolar spectrum disorder. Can J Psychiatry. 2002;47(2):125-34.

17. Dias VV, Brissos S, Carita Al. Clinical and neurocognitive correlates of insight in patients with bipolar I disorder in remission. Acta Psychiatr Scand. 2008b;117(1):28-34. 
18. Güçlü 0, Karaca 0, Yildirim B, Ozköse MM, Erkiran M. The relationship between insight and clinical features in bipolar disorder. Turk J Psychiatry. 2011;22(4):1-7.

19. Amanzio M, Vase L, Leotta D, Miceli R, Palermo S, Geminiani G. Imparied awareness of deficits in Alzheimer disease: the role of everyday dysfunction. J Int Neuropsych Soc. 2013;19('):63-72.

20. Camelo EVM, Netto T, Velasques B, Ribeiro P, Cheniaux E. Attention impairment in bipolar disorder: a systematic review. Psychol Neurosci. 2013;6(3):299-309.

21. Vohringer PA, Barroilhet SA, Amerio A, Reale ML, Alvear K, Vergne D, et al. Cognitive impairment in bipolar disorder and schizophrenia: a systematic review. Front Psychiatry. 2013:4(87):1-11.

22. Pini S, De Queiroz V, Dell'Osso L, Abelli M, Mastrocinque C, Saettoni M, et al. Cross-sectional similarities and differences between schizophrenia, schizoaffective disorder and mania or mixed mania with mood-incongruent psychotic features. Eur Psychiatry. 2004;19(1):8-14.

23. Colis MJ, Steer RA, Beck AT. Cognitive insight in inpatients with psychotic, bipolar and major depressive disorders. J Psychopathol Behav Assess. 2006;28(4):242-9.

24. Braw Y, Sitman R, Sela T, Erez G, Bloch Y, Levkovitz Y. Comparison of insight among schizophrenia and bipolar disorder patients in remission of affective and positive symptoms: analysis and critique. Eur Psychiatry. 2012;27(8):612-8.

25. Calatayud GL, Sebastián NH, García-iturrospe EA, Piqueras JCG, Arias JS, Cercós CL. Relationship between insight, violence and diagnoses in psychotic patients. Rev Psiquiatr Salud Ment. 2012;5(1):43-7.

26. Amador XF, Strauss DH, Yale S, Flaum MM, Endicott J, Gorman JM. Assessment of insight in psychosis. Am J Psychiatry. 1993;150(6):873-9.

27. David AS. Insight and psychosis. Br J Psychiatry. 1990;156:798-808.

28. Kemp R, David A. Insight and compliance. In: Blackwell B, editor. Insight and compliance. Newark (NJ): Gordon and Breach Publishing Group; 1996. p. 61-84.

29. Fennig S, Everett E, Bromet EJ, Jandorf L, Fennig SR, Tenenberg-Karant M, et al. Insight in first-admission psychotic patients. Schizophr Res. 1996;22(3):257-63.

30. Michalakeas A, Skoutas C, Charalambous A, Peristeris A, Marinos V, Keramari E, et al. Insight in schizophrenia and mood disorders and its relation to psychopathology. Acta Psychiatr Scand. 1994;90(1):46-9.

31. Yen CF, Chen CS, Yeh ML, Yen JY, Ker JH, Yang SJ. Comparison of insight in patients with schizophrenia and bipolar disorder in remission. J Nerv Ment Dis. 2002b;190(2):847-9.

32. Daneluzzo E, Arduini L, Rinaldi O, Di Domenico M, Petruzzi C, Kalyvoka A, et al. PANSS factors and scores in schizophrenic and bipolar disorders during an index acute episode: a further analysis of the cognitive component. Schizophr Res. 2002;56(1-2):129-36.

33. Young DA, Zakzanis KK, Bailey C, Davila R, Griese J, Sartory G, et al. Further parameters of insight and neuropsychological deficit in schizophrenia and other chronic disease. J Nerv Ment Dis. 1998;186(1):44-50

34. Govil S, Sengupta SN, Sharma P, Kumar D. A study of comparison of insight in patients with schizophrenia and bipolar affective disorder in remission phase. Abstracts for Poster Session I. Eur Psychiatry. 2008;23:S81-191.

35. Masson M, Azorin JM, Bourgeois ML. La conscience de la maladie dans les troubles schizophréniques, schizo-affectifs, bipolaires et unipolaires de l'humeur: résultats d'une étude comparative de 90 patients hospitalisés. Ann Med Psychol. 2001;159:369-74.

36. Varga M, Magnusson A, Flekkoy K, David AS, Opjordsmoen S. Clinical and neuropsychological correlates of insight in schizophrenia and bipolar I disorder: does diagnosis matter? Compr Psychiatry. 2007;48(6):583-91.

37. Jonsdottir H, Opjordsmoen S, Birkenaes AB, Simonsen C, Engh JA, Ringen PA, et al. Predictors of medication adherence in patients with schizophrenia and bipolar disorder. Acta Psychiatr Scand. 2013;127(1):23-33

38. Arduini L, Kalyvoka A, Stratta P, Rinaldi O, Daneluzzo E, Rossi A. Insight and neuropsychological function in patients with schizophrenia and bipolar disorder with psychotic features. Can J Psychiatry. 2003;48(5):338-41.

39. Williams $\mathrm{CC}$, Collins A. Factors associated with insight among outpatients with serious mental illness. Psychiatr Serv. 2002;53(1):96-8.

40. Weiler MA, Fleisher MH, McArthur-Campbell D. Insight and symptom change in schizophrenia and other disorders. Schizophr Res. 2000;45(1-2):29-36.

41. Ghaemi SN, Boiman E, Goodwin FK. Insight and outcome in bipolar, unipolar and anxiety disorders. Compr Psychiatry. 2000b;41(3):167-71
42. Dell'Osso L, Pini S, Cassano GB, Mastrocinque C, Seckinger RA, Saettoni M, et al. Insight into illness in patients with mania, mixed mania, bipolar depression and major depression with psychotic features. Bipolar Disord. 2002;4(5):315-22.

43. Pallanti S, Quercioli L, Pazzagli A, Rossi A, Dell'Osso L, Pini S, et al. Awareness of illness and subjective experience of cognitive complaints in patients with bipolar I and bipolar II disorder. Am J Psychiatry. 1999;156(7):1094-6.

44. Cassidy F. Insight in bipolar disorder: relationship to episode subtypes and symptom dimensions. Neuropsychiatr Dis Treat. 2010;6:627-31.

45. Yen $\mathrm{CF}_{\text {, }}$ Cheng $C \mathrm{CP}_{\text {, }} \mathrm{K}_{0} \mathrm{CH}$, Yen JY, Huang CF, Chen CS. Relationship between insight and neurocognition in patients with bipolar I disorder in remission. Compr Psychiatry. 2008a;49(4):335-9.

46. Yen $\mathrm{CF}_{\text {, Chen }} \mathrm{CS}$, Yeh ML, Ker JH, Yang SJ, Yen JY. Correlates of insight among patients with bipolar I disorder in remission. J Affect Disord. 2004;78(1):57-60.

47. Dias VV, Brissos S, Frey BN, Kapczinski F. Insight, quality of life and cognitive functioning in euthymic patients with bipolar disorder. J Affect Disord. 2008a;110(1-2):75-83.

48. Acosta FJ, Veja D, Torralba L, Navarro S, Ramallo-Fariña Y, Fiúza D, et al. Hopeleness and suicidal risk in bipolar disorder: a study in clinically nonsyndromal patients. Compr Psychiatry. 2012;53(8):1103-9.

49. Bressi C, Porcellana M, Marinaccio PM, Nocito EP, Ciabatti M, Magri L, et al. The association between insight and symptoms in bipolar inpatients: an Italian prospective study. Eur Psychiatry. 2012;27(8):619-24.

50. Van Der Werf-Eldering MJ, Van der Meer L, Burger H, Holthausen EAE, Nolen WA, Aleman A. Insight in bipolar disorder: associations with cognitive and emotional processing and illness characteristics. Bipolar Disord. 2011:13(4):343-54.

51. Gonzalez VM. Recognition of mental illness and suicidality among individuals with serious mental illness. J Nerv Ment Dis. 2008;196(10):727-34.

52. Yen CF, Cheng CP, Ko CH, Yen JY, Huang CF, Chen CS. Suicidality and its association with insight and neurocognition in Taiwanese patients with bipolar I disorder in remission. J Nerv Ment Dis. 2008c;196(6):462-7.

53. Pini S, Dell'Osso L, Amador XF, Mastrocinque C, Saettoni M, Cassano GB. Awareness of illness in patients with bipolar I disorder with or without comorbid anxiety disorders. Aust N Z J Psychiatry. 2003;37(3):355-61.

54. Lam D, Wong G. Prodromes, coping strategies, insight and social functioning in bipolar affective disorders. Psychol Med. 1997;27(5):1091-100.

55. Ghaemi SN, Hebben N, Stoll AL, Pope HG. Neuropsychological aspects of lack of insight in bipolar disorder: a preliminary report. Psychiatry Res. 1996;65(2):113-20.

56. Cerit C, Filizer A, Tural Ü, Tufan AE. Stigma: a core factor on predicting functionality in bipolar disorders. Compr Psychiatry. 2012;53(5):484-9.

57. González-Ortega I, Mosquera F, Echeburúa E, González-Pinto A. Insight, psychosis and aggressive behaviour in mania. Eur J Psychiatry. 2010;24(2):70-7.

58. Varga M, Babovic A, Flekkoy K, Ronneberg U, Landro NI, David AS, et al. Reduced insight in bipolar I disorder: neurofunctional and neurostructural correlates: a preliminar study. J Affect Disord. 2009;116(1-2):56-63.

59. Yen CF, Chen CS, Tem JY, Ko CH. The predictive effect of insight on adverse clinical outcomes in bipolar I disorder: a two-year prospective study. J Affect Disord. 2008b;108(1-2):121-7.

60. Yen $\mathrm{CF}_{\text {, }} \mathrm{Chen} \mathrm{CC}_{\text {, Cheng }} \mathrm{CP}_{\text {, Yen } \mathrm{CN}}$, Lin HC, $\mathrm{Ko}$ CH, et al. Comparisons of insight in schizophrenia, bipolar I disorder, and depressive disorders with and without comorbid alcohol use disorder. Psychiatry Clin Neurosci. 2008d;62(6):685-90.

61. Yen CF, Cheng CP, Huang CF, Yen JY, Ko CH, Chen CS. Quality of life and its association with insight, adverse effects of medication and use of atypical antipsychotics in patients with bipolar disorder and schizophrenia in remission. Bipolar Disord. 2008e;10(5):617-24.

62. Yen CF, Chen CS, Yang SJ, Ko CH, Yen JY, Huang CF. Relationships between insight and psychosocial adjustment in patients with bipolar I disorder. Bipolar Disord. 2007b;9(7):737-42.

63. Schuepbach D, Goetz I, Boeker H, Hell D. Voluntary vs involuntary hospital admission in acute mania of bipolar disorder: results from the Swiss sample of the EMBLEM study. J Affect Disord. 2006:90(1):57-61.

64. Varga M, Magnusson A, Flekkoy K, Ronneberg U, Opjordsmoen S. Insight, symptoms and neurocognition in bipolar I patients. J Affect Disord. 2006;91:1-9.

65. Yen CF, Chen CS, Yeh ML, Yang SJ, Ker JH, Yen JY. Changes of insight in maniac episodes and influencing factors. Compr Psychiatry. 2003;44(5):404-8. 
66. Peralta V, Cuesta MJ. Lack of insight in mood disorders. J Affect Disord. 1998;49(1):55-8.

67. Yen CF, Chung LC, Chen CS. Insight and neuropsychological functions in bipolar outpatients in remission. J Nerv Ment Dis. 2002a;19(10):713-5.

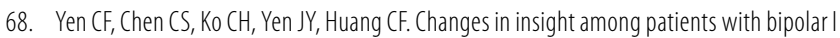
disorder: a 2-year prospective study. Bipolar Disord. 2007a;9(3):238-42.

69. Cassidy F, McEvoy JP, Yang YK, Wilson WH. Insight is greater in mixed than in pure manic episodes of bipolar I disorder. J Nerv Ment Dis. 2001;189(6):398-9.

70. Rossi A, Daneluzzo E, Arduini L, Di Domenico M, Stratta P, Petruzzi C. Cognitive symptoms of mania in pure and mixed episodes evaluated with the Positive and Negative Syndrome Scale. Eur Arch Psychiatry Clin Neurosci. 2000;250(5):254-6.

71. Dell'Osso L,Pini S, Tundo A, Sarno N, Musetti L, Cassano GB. Clinical characteristics of mania, mixed mania, and bipolar depression with psychotic features. Compr Psychiatry. 2000;41(4):242-7.

72. Sims A. Symptoms in the mind: an introduction to descriptive psychopathology. 3rd ed. London: Saunders; 2006.

73. Belfort T, Sousa MFB, Santos RL, Lima M, Torres B, Dias R, et al. Consciência do déficit na doença de Alzheimer: diferenças entre os domínios cognitivo e funcional. J Bras Psiquiatr. 2013:62(3):217-24

74. Dourado MCN, Laks J, Rocha M, Soares C, Leibing A, Engelhardt E. Consciência da doença na demência: resultados preliminares em pessoas com doença de Alzheimer leve e moderada. Arq Neuropsiquiatr. 2005;63(1):114-8.

75. Insel TR, Akiskal HS. Obsessive-compulsive disorder with psychotic features: a phenomenologic analysis. Am J Psychiatry. 1986;143(12):1527-33.
76. Spitznagel MB, Tremont G, Brown LB, Gunstad J. Cognitive reserve and the relationship between depressive symptoms and awareness of deficits in dementia. J Neuropsych Clin N. 2006;18(2):186-90

77. Ravi Kishore V, Samar R, Janardhan Reddy YC, Chandrasekhar CR, Thennarasu K. Clinical characteristics and treatment response in poor and good insight obsessive-compulsive disorder. Eur Psychiatry. 2004;19(4):202-8.

78. Tumkaya S, Karadag F, Oguzhanoglu NK, Tekkanat C, Varma G, Ozdel 0, et al. Schizophrenia with obsessive-compulsive disorder and obsessive-compulsive disorder with poor insight: a neuropsychological comparison. Psychiatry Res. 2009;165(1-2):38-46.

79. Leicht H, Berwig M, Gertz HJ. Anosognosia in Alzheimer's disease: the role of impairment levels in assessment in insight across domains. J Int Neuropsych Soc. 2010;16(3):463-73.

80. Stewart G, McGeown WJ, Shanks MF, Venneri A. Anosognosia of memory impairment in Alzheimer's disease. Acta Neuropsychiatr. 2010;22(4):180-7.

81. Catapano F, Sperandeo R, Perris F, Lanzaro M, Maj M. Insight and resistance in patients with obsessive compulsive disorder. Psychopathology. 2001;34(2):62-8.

82. Cheniaux E, Landeira-Fernandez J, Telles LL, Lessa JLM, Dias A, Duncan T, et al. Does schizoaffective disorder really exist? A systematic review of the studies that compared schizoaffective disorder with schizophrenia or mood disorders. J Affect Disord. 2008;106(3):209-17.

83. Silva RA, Mograbi DC, Silveira LAS, Nunes ALS, Novis FD, Landeira-Fernandez J, et al. The reliability of self-assessment of affective state in different phases of bipolar disorder. J Nerv Ment Dis. 2014;202(5):1-5. 